\title{
A biocompatible fluorescent probe for the selective detection of amyloid fibrils
}

\author{
Anirban Das, ${ }^{[a]}$ Tanoy Dutta,${ }^{[a]}$ Laxmikant Gadhe, ${ }^{[b]}$ Apurba Lal Koner, ${ }^{*[a]}$ and Ishu Saraogi ${ }^{*[a] ~[c]}$
}

[a] Department of Chemistry, Indian Institute of Science Education and Research Bhopal, Bhopal Bypass Road, Bhauri, Bhopal 462066, MP, India [b] Department of Biosciences and Bioengineering, Indian Institute of Technology, Powai, Bombay 400076, India, [c] Department of Biological Sciences, Indian Institute of Science Education and Research Bhopal, Bhopal Bypass Road, Bhauri, Bhopal 462066, MP, India

Correspondence to ishu@iiserb.ac.in, akoner@iiserb.ac.in

\begin{abstract}
The misfolding and aggregation of proteins leading to amyloid formation has been linked to numerous diseases, necessitating the development of tools to monitor the fibrillation process. Here we report an intramolecular charge transfer (ICT) dye, DMNDC, as an alternative to Thioflavin-T (ThT), most commonly used for monitoring amyloid fibrils. Using insulin as a model protein, we show that DMNDC efficiently detects all stages of fibril formation, namely, nucleation, elongation, and saturation. An approximately $70 \mathrm{~nm}$ hypsochromic shift along with a large increase in emission intensity was observed upon binding of DMNDC to protein fibrils. The aggregation kinetics of insulin remained unaffected at excess DMNDC concentration, suggesting that DMNDC does not inhibit insulin aggregation. Additionally, the efficient cellular internalization and low toxicity of DMNDC make it highly suited for sensing and imaging of amyloid fibrils in the complex biological milieu.
\end{abstract}

\section{INTRODUCTION}

Amyloids are extracellular, insoluble protein deposits characterized by a cross $\beta$-sheet structure. ${ }^{1}$ The misfolding and aggregation of proteins leading to amyloid formation are associated with several pathological conditions including Alzheimer's disease, Parkinson's disease, and type-II diabetes. ${ }^{2-4}$ Thus, there is an urgent need to develop methods for the detection of fibril formation both in vitro and in vivo. Although various spectroscopic and microscopic techniques have been used to study amyloidogenesis, ${ }^{5,6}$ fluorescence spectroscopy is considered to be the method of choice owing to its simplicity, rapidity, and reliability. ${ }^{7-11}$ The use of intrinsic fluorescence of the aggregating proteins, via monitoring of tyrosine or tryptophan fluorescence, ${ }^{12}$ has not been very successful as these fluorophores have inherently weak blue emission. Therefore, it is of utmost importance to develop extrinsic reporter fluorophores to monitor protein fibrillation.

To date, thioflavin-T (ThT) has been the fluorophore of choice to monitor in vitro protein fibrillation. ${ }^{13-15}$ Fibril-bound ThT displays a significant increase in fluorescence intensity compared to free ThT, as it becomes conformationally rigid on amyloid binding. The high specific interaction of ThT with amyloid fibrils makes it a sensitive tool for studying fibrillation kinetics. Despite its wide use in detecting amyloid fibrils, ThT has various limitations such as small Stokes shift, inability to report on oligomeric intermediates, and potential for artefacts when studying the fibrillation kinetics in the presence of small molecule inhibitors. ${ }^{16,17,18}$ In recent years, researchers have synthesized various fluorophores for the improved detection of amyloid fibrils. ${ }^{16,19-21}$ However, their tedious synthesis and high cost limit their use as amyloid markers.

Imperiali and co-workers first reported 6-N,N-dimethylamino2,3-naphthalimide (6-DMN) for probing protein-protein interactions. ${ }^{22,23}$ Later, Koner group showed that the diester
A
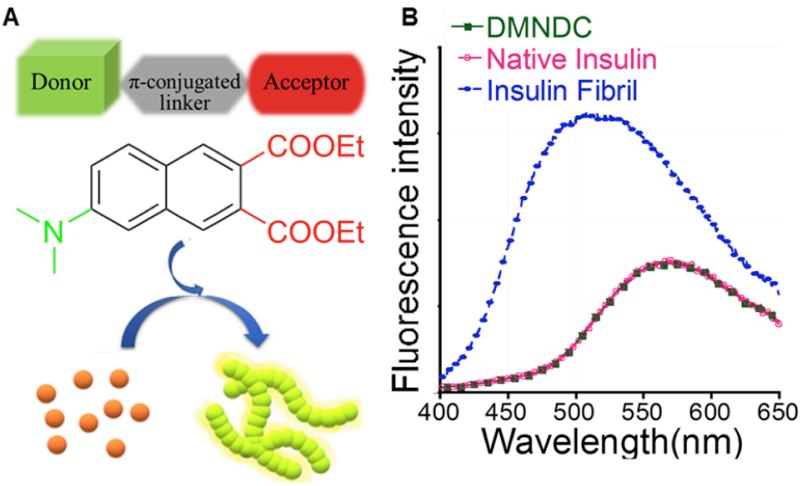

Figure 1. A A) Schematic showing the ICT nature of DMNDC and its interaction with insulin fibrils. B) Detection of insulin fibrillation by DMNDC at $\mathrm{pH} 7.4\left(\lambda_{\mathrm{ex}}=338 \mathrm{~nm}\right)$. The emission maxima of DMNDC is at $566 \mathrm{~nm}$ for native insulin, and at $490 \mathrm{~nm}$ for insulin fibrils.

derivative of 6-DMN, diethyl 6-(dimethylamino) naphthalene2,3-dicarboxylate (DMNDC, Fig. 1A) exhibits excellent solvatochromic properties, and can report on self-assembling processes and micro-environments of different micelles. ${ }^{24}$ DMNDC contains an electron-rich donor site $(\mathrm{N}, \mathrm{N}$-dimethyl amine unit), which is connected to two electron-withdrawing acceptor units (carboxylate esters), through conjugation via a naphthalene spacer (Fig. 1A). Due to its ability to detect the hydrophobic pockets of bovine serum albumin (BSA), ${ }^{24}$ we reasoned that DMNDC might be able to distinguish the native state of proteins from their amyloid state.

Insulin is a 51-amino acid polypeptide hormone that readily undergoes fibrillation, and has been widely used as a model 
protein to study fibril formation. ${ }^{25}$ Insulin, which is routinely prescribed to manage the symptoms of diabetes, has been reported to form fibrils at the site of repeated injection in diabetic patients. ${ }^{4}$ High concentrations of insulin encountered during its industrial production and transportation also lead to insulin fibrillogenesis, ${ }^{26}$ which is further exacerbated at high temperature, low $\mathrm{pH}$ and increased ionic strength. Although the exact molecular nature of the interactions that lead to fibril formation is unknown, it is recognized that insulin monomers undergo partial unfolding, which associate to form $\beta$-sheet rich fibrils. ${ }^{27}$

Herein, a small intramolecular charge transfer dye (ICT) DMNDC was used as an ex-situ fluorophore for the detection of amyloid fibrils using insulin as a model protein (Fig. 1). Our results demonstrated that DMNDC can successfully detect different stages of fibril formation including nucleation, elongation, and saturation. Unlike the native form of the protein, insulin fibrils induced a large $(\sim 70 \mathrm{~nm})$ blue shift in the emission maxima of DMNDC. Moreover, DMNDC did not show any significant fluorescence enhancement in the presence of several native proteins, confirming its specificity towards amyloid fibrils. Cell-based assays showed efficient internalization, and localization of DMNDC in the mitochondria, which could be utilized to investigate mitochondrial dysfunction associated with Alzheimer's disease.

\section{RESULTS AND DISCUSSION}

DMNDC was synthesized as previously reported ${ }^{20}$ and tested for its ability to detect insulin fibrils. DMNDC was incubated either with native insulin solution or insulin solution after complete fibrillation was achieved. Upon irradiation at $338 \mathrm{~nm}$, DMNDC alone or with native insulin displayed similar fluorescence emission profiles with the emission maximum at $566 \mathrm{~nm}$. The emission maximum shifted to $490 \mathrm{~nm}$ upon addition of fibrils, resulting in a $\sim 10$-fold increase in fluorescence intensity at $490 \mathrm{~nm}$ (Fig. 1B). The specific recognition of insulin fibrils over native insulin indicated the potential utility of this fluorophore as an amyloid marker.

We next compared the ability of DMNDC to detect fibrils at three different $\mathrm{pH}$ values: $\mathrm{pH} 7.4$ to mimic the physiological $\mathrm{pH}$, 4.0 which is close to the pl value of insulin, and 1.6 as insulin aggregation is often carried out at acidic $\mathrm{pH}$ in vitro. ${ }^{28}$

At $\mathrm{pH} 4.0$, DMNDC behaved in a manner identical to $\mathrm{pH} 7.4$ (Fig. 2A-B). In contrast, the fluorescence of DMNDC was quenched at $\mathrm{pH} 1.6$ likely due to protonation of the nitrogen atom of the dimethylamine unit. This observation is consistent with a previous report that the $\mathrm{pK}_{a}$ of the $\mathrm{N}, \mathrm{N}$-dimethyl amine unit is $2.65 .^{24}$ The observed blue shift at $\mathrm{pH} 4.0$ and above can be attributed to the binding of DMNDC to hydrophobic patches formed upon protein fibrillation. ${ }^{24}$
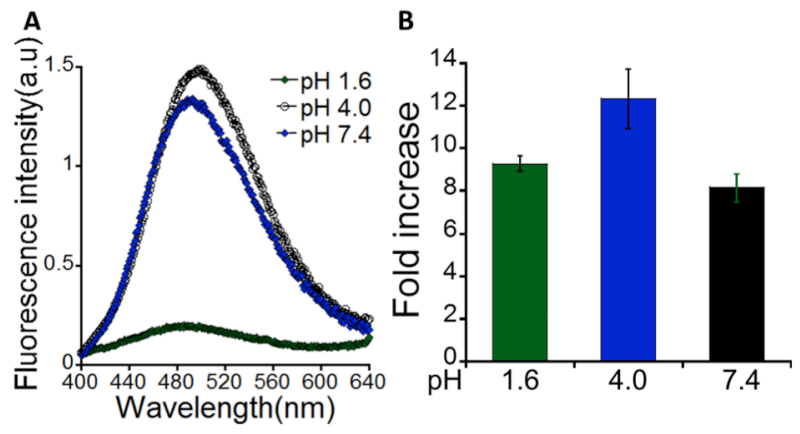

Figure 2. A) Detection of insulin fibrillation by DMNDC at different $\mathrm{pH}$ values. B) Relative change in fluorescence intensity of DMNDC (15 $\mu \mathrm{M})$ bound to fibrillated insulin $(3.75 \mu \mathrm{M})$ at $490 \mathrm{~nm}$ with respect to the free DMNDC at the specified $\mathrm{pH}$.
The successful detection of insulin amyloids prompted us to test whether DMNDC can be used to monitor the kinetics of insulin fibrillation. From an insulin solution fibrillated at $\mathrm{pH} 7.4$, aliquots were removed at different time intervals, mixed with DMNDC, and subjected to fluorescence measurement. We found that DMNDC authentically reported on the nucleation, elongation, and saturation stages of fibril formation analogous to ThT (Fig. 3A). A cyan blue fluorescence was observed when the DMNDC containing fibrillated insulin sample was exposed to UV light, unlike control samples lacking either insulin fibrils or DMNDC (Fig. 3B). Under visible light, no difference in the three samples was observed, indicating that the dye was fluorescent only under UV irradiation. The formation of fibrils was further confirmed by TEM imaging (Fig. 3C), and confocal microscopy of the fibrils in the presence of DMNDC (Fig. 3D).

Further, we tested whether the fluorescence of DMNDC increased proportionately with fiber concentration, and determined the lowest fibril concentration that could be detected by DMNDC. For this experiment, DMNDC $(20 \mu \mathrm{M})$ was mixed with different concentrations of fibrillated insulin (0$50 \mu \mathrm{M})$, and the fluorescence was recorded. We observed a gradual linear increase in DMNDC fluorescence with an increase in fibrillated insulin concentration until $50 \mu \mathrm{M}$, the highest concentration used in our study. (Fig. 4A, Fig. S4A). DMNDC was able to clearly detect the fibrils formed from native insulin at a concentration as low as $5-10 \mu \mathrm{M}$. A control experiment performed with different concentrations of native
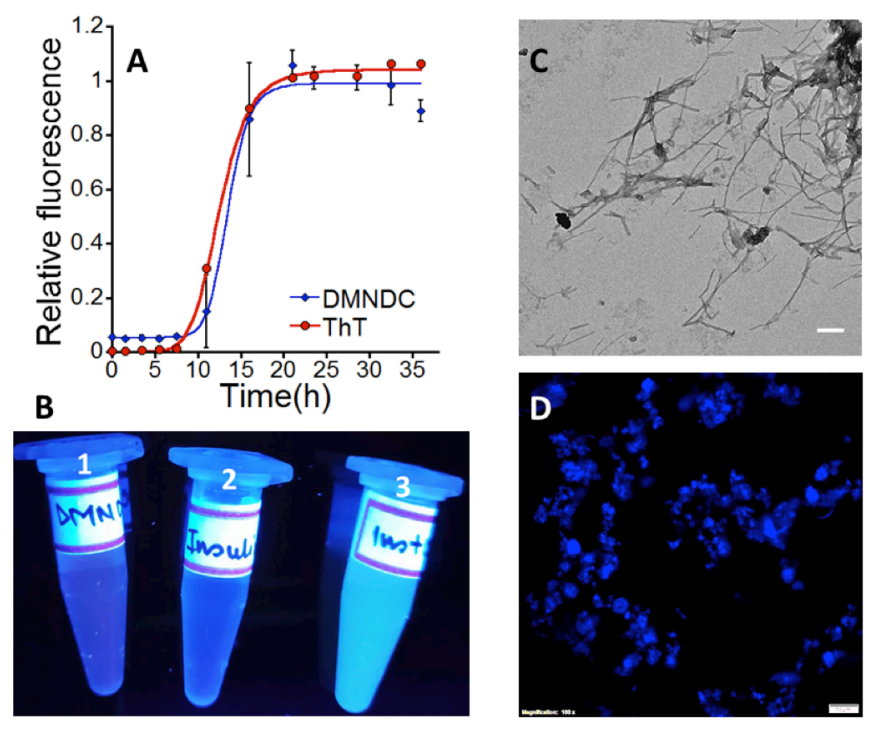

Figure 3. A) Fibrillation kinetics of insulin at $\mathrm{pH} 7.4$ as detected by DMNDC and ThT. B) Image showing DMNDC fluorescence emission up on interaction with insulin fibrils under UV light. 1-only DMNDC; 2-Aggregated insulin solution only; 3-Aggregated insulin solution incubated with DMNDC. C) TEM image of the insulin fibrils (Scale: $200 \mathrm{~nm}$ ) D) Confocal microscopy image of insulin fibers in the presence of DMNDC (Scale: $10 \mu \mathrm{m}$ ).

insulin showed no significant enhancement in DMNDC fluorescence. Next, we wondered whether DMNDC could interfere with the protein fibrillation process. To verify this, 10 $\mu \mathrm{M}$ insulin was incubated with a 2-fold excess of DMNDC (20 $\mu \mathrm{M})$, and the fibrillation of insulin was monitored using ThT. DMNDC containing insulin showed no significant difference in the fibrillation kinetics compared to a control sample containing insulin only. This result suggested that DMNDC does not interfere with the fibrillation kinetics, and thus can be used as a dye for detection of amyloids (Fig. 4B).

To check whether DMNDC specifically bound to fibrils, DMNDC $(30 \mu \mathrm{M})$ was incubated with insulin fibers $(20 \mu \mathrm{M})$, or several native proteins including lysozyme, $\alpha$-synuclein, and insulin. 

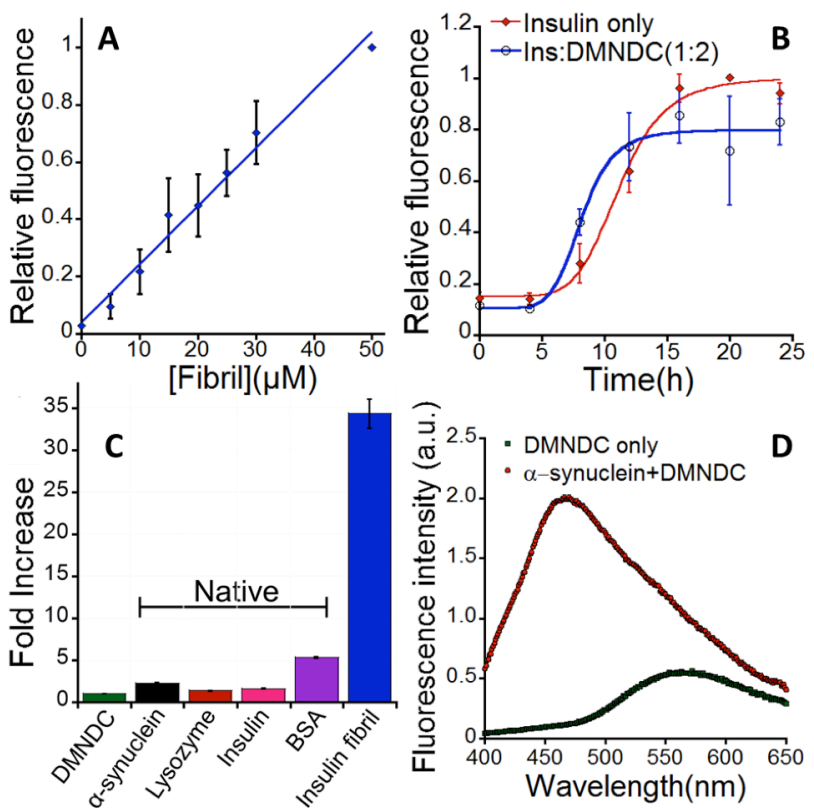

Figure 4. A) Increase in fluorescence intensity of DMNDC $(20 \mu \mathrm{M})$ with increasing concentrations of fibrillated insulin. The data were normalized with respect to fluorescence intensity of DMNDC at $50 \mu \mathrm{M}$ fibrillated insulin. B) Kinetics of insulin $(10 \mu \mathrm{M})$ fibrillation in the absence and presence of DMNDC $(20 \mu \mathrm{M})$ measured using ThT fluorescence. C) The increase in fluorescence intensity of DMNDC $(30 \mu \mathrm{M})$ at $490 \mathrm{~nm}$ with the native form of several proteins compared to insulin fibrils (30 $\mu \mathrm{M}$ each). D) Detection of $\alpha$-synuclein aggregates $(20 \mu \mathrm{M})$ by DMNDC $(30 \mu \mathrm{M})$.

The blue shift of the emission maximum and increase in DMNDC fluorescence emission intensity was observed only in the case of insulin fibers (Fig. 4C), suggesting that DMNDC specifically reports only on amyloid fibrillar structures. We further checked whether DMNDC was a specific reporter for insulin amyloid or it could detect other amyloid proteins. Our data show that DMNDC could also successfully recognize the fibrillar form of $\alpha$-synuclein, but not the native form of the protein (Fig. 4D). This suggests that DMNDC could be a generic amyloid sensor and can potentially be used as an amyloid specific biomarker.

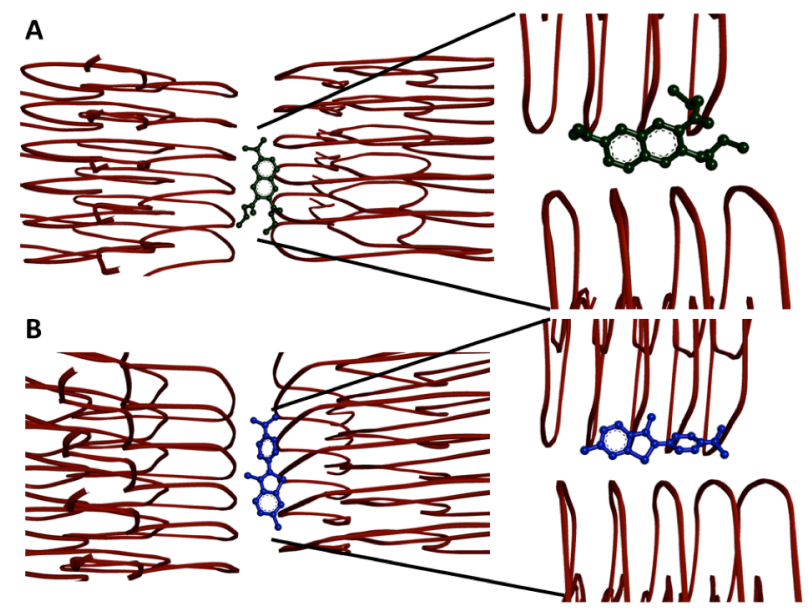

Figure 5. Molecular docking of (A) DMNDC and (B) ThT to $\alpha$-synuclein fibrils (PDB ID: 6A6B). The brown lines represent $\beta$-sheet fibrils of $\alpha$ synuclein. DMNDC is marked in green and ThT in blue respectively.
We carried out molecular docking studies to rationalize the binding mode of DMNDC to the fibrils. A recently reported $\alpha$ synuclein fibril structure was chosen as the representative fibril model for docking (PDB ID: 6A6B). ${ }^{29}$ Amyloid fibrils are characterized by long, unbranched ribbon-like morphology, with a cross- $\beta$ structure running perpendicular to the fibrillar axis. ${ }^{30}$ DMNDC was found to bind parallel to the fibrillar axis as depicted in Fig. 5A. The docked pose was strikingly similar to that obtained with ThT (Fig. 5B), and was in agreement with reported ThT-fibril binding. ${ }^{14}$

As DMNDC acts as a turn-on probe for detecting amyloid fibrils, it is interesting to observe the localization of the probe in living cells. The subcellular localization of DMNDC in live-cells was studied using confocal microscopy (Fig. 6). The co-localization experiment was performed in live baby hamster kidney (BHK21) cells with DMNDC and commercially available Mito-Tracker Red. This experiment confirmed that DMNDC localizes to the mitochondria with a Pearson's coefficient of $0.94 \pm 0.03$. Control experiments with Lysotracker Red and ER tracker Red confirmed that the probe did not localize in the lysosome or ER. Further, DMNDC showed high photostability, and low cytotoxicity with an $\mathrm{IC}_{50}>100 \mu \mathrm{M}$ in an MTT assay (Fig. 6).

These results are significant because recent investigations suggest that intracellular accumulation of $\mathrm{A} \beta$ may be an early event in the pathogenesis of Alzheimer's disease and Down syndrome. ${ }^{31,32}$ In particular, $\beta$-amyloids $(\mathrm{A} \beta)$ target mitochondria and accumulate in the mitochondrial import channels. ${ }^{33}$ Mitochondrial $A \beta$ plays a key role in the alteration of the mitochondrial dynamics by interacting with several proteins inside the organelle. ${ }^{34}$ The ability of DMNDC to localize to the mitochondria may be valuable to study $\mathrm{A} \beta$ aggregation events inside the mitochondria.

In conclusion, we propose that DMNDC is an excellent probe to detect the various stages of protein fibrillation. The probe emits a strong blue fluorescence upon binding to insulin fibrils. Importantly, the Stokes shift observed upon binding to insulin fibrils confers a significant advantage over ThT, which does not exhibit such a shift. Docking studies suggest that DMNDC binds parallel to the fibrillar axis analogous to ThT. Furthermore, as DMNDC localizes to the mitochondria, it can be used as a probe to study mitochondrial dysfunction in Alzheimer's disease pathogenesis, which is induced by $\beta$ amyloid accumulation. The low cytotoxicity and efficient subcellular localization of DMNDC lends itself well to in vivo applications. We believe that DMNDC can be an excellent

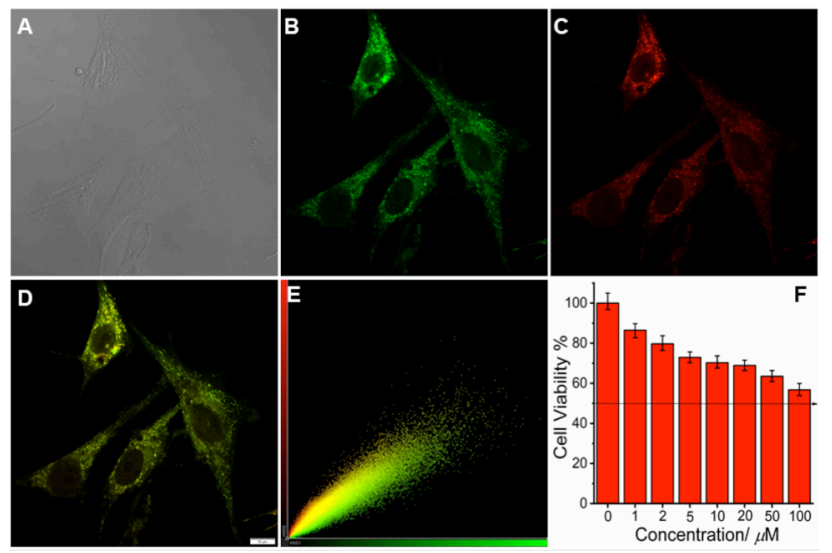

Figure 6. Live-cell confocal microscopy images showing the subcellular localization of DMNDC in BHK-21 cells. A) DIC image, B) cells stained with $10 \mu \mathrm{M}$ of DMNDC, C) cells stained with $300 \mathrm{nM}$ of MitoTracker Red D) merge of B) and C); E) Scatter plot with Pearson's co-efficient $0.94 \pm 0.03$, F) MTT assay showing cell viability in the presence of DMNDC $(0-100 \mu \mathrm{M})$. 
probe for amyloid, and enriches the library of probes to detect amyloidosis.

\section{MATERIALS AND METHODS}

Materials. $\mathrm{KH}_{2} \mathrm{PO}_{4}$ and $\mathrm{K}_{2} \mathrm{HPO}_{4}$ were obtained from Alfa Aesar, $\mathrm{NaCl}$ and $\mathrm{HCl}$ (conc.) from Sigma Aldrich. Recombinant human insulin was purchased from MP Biomedicals. Lysozyme and BSA were purchased from Sigma Aldrich; $\alpha$-synuclein was a kind gift from Prof. Samir Maji, IIT Bombay.

Sample Preparation. A stock solution of insulin was prepared by dissolving insulin in $20 \mathrm{mM}$ PBS (pH 7.4) unless specified otherwise. For $\mathrm{pH} 1.6$ and 4.0 , aqueous $\mathrm{HCl}$ solution containing $100 \mathrm{mM} \mathrm{NaCl}$ was used to solubilize the insulin.

Insulin Fibrillation. The insulin stock solution was diluted to a concentration of $15 \mu \mathrm{M}$, and incubated at $37^{\circ} \mathrm{C}$, with agitation to achieve fibrillation. For studying fibrillation kinetics using DMNDC, insulin solution was diluted to $22.5 \mu \mathrm{M}$ in $20 \mathrm{mM}$ PBS buffer and agitated for fibrillation. In all experiments, the insulin fibril concentration was determined to be the maximum soluble insulin concentration used, assuming $100 \%$ fibril formation. To measure DMNDC fluorescence during fibrillation kinetics, a stock solution of DMNDC $(20 \mu \mathrm{M})$ was prepared in PBS buffer (pH 7.4). At specified time intervals, an aliquot of the aggregating insulin solution was removed, added to DMNDC solution $\left(15 \mu \mathrm{M}\right.$ final) and DMNDC fluorescence $\left(\lambda_{\mathrm{ex}}=338 \mathrm{~nm}\right.$, $\lambda_{\text {mon }}=400-650 \mathrm{~nm}$ ) was measured using HORIBA Fluorolog-3 Spectrophotometer.

a-synuclein aggregation measurement using DMNDC fluorescence. Low molecular weight (LMW) a-synuclein was prepared as described. ${ }^{35}$ Briefly, $10 \mathrm{mg}$ of lyophilized $\alpha$ synuclein powder was dissolved in $20 \mathrm{mM}$ phosphate buffer saline (PBS). A few microliters of $1 \mathrm{M} \mathrm{NaOH}$ was added to completely solubilize the protein, and the $\mathrm{pH}$ of the solution was adjusted to 7.4. The resulting solution was centrifuged at 10000 rpm for 20 minutes at $4^{\circ} \mathrm{C}$ and the supernatant was transferred to a $10 \mathrm{KDa}$ MWCO mini dialysis unit (Thermo Scientific). The protein was dialyzed for $12 \mathrm{~h}$ at $4^{\circ} \mathrm{C}$, and the resulting solution was filtered using a $100 \mathrm{KDa}$ cut off filter (Amicon). The flowthrough containing LMW $\alpha$-synuclein was collected. The stock solution was diluted to $400 \mu \mathrm{M}$, and incubated at $37^{\circ} \mathrm{C}$, with agitation to achieve fibrillation. At the completion of fibrillation, $20 \mu \mathrm{M}$ aggregated $\alpha$-synuclein solution was mixed with $30 \mu \mathrm{M}$ dye and the fluorescence was measured.

Molecular Docking. Molecular docking was carried out to determine the predicted mode of binding of DMNDC and ThT to a-synuclein fibrils using Auto dock $4.0 .^{36}$ The fibrillar structure of a-synuclein, PDB 6A6B, was used for the docking. ${ }^{37}$ Blind docking, with no preference for ligand binding site, was performed. Gasteiger charges were applied to both protein and the ligands. DMNDC and ThT were both docked against the surface of fibrils. In the docking analysis, $\alpha$-synuclein fibrillar structure was treated as a rigid body. All other parameters were set to default during the docking process. The results were analyzed and figures were prepared using Discovery Studio Visualizer Software, Version 4.0 (2012) ${ }^{38}$

Culture Method. BHK-21 cells were obtained from NCCS, Pune, India and were grown in a $25 \mathrm{~cm}^{2}$ cell culture flask (Corning, USA) using DMEM (phenol red free) containing $10 \%$ (v/v) FBS and $1 \%(\mathrm{v} / \mathrm{v})$ antibiotic cocktail in $5 \% \mathrm{CO}_{2}$ at $37^{\circ} \mathrm{C}$ in a $\mathrm{CO}_{2}$ incubator. For imaging purposes, cells were grown to $75 \%-80 \%$ confluency in the $35 \mathrm{~mm}$ glass-bottom imaging dishes (170 $\pm 5 \mu \mathrm{m}$, Ibidi, Germany) in DMEM with 10\% FBS. The cells were washed twice with PBS (pH 7.4) containing 5 $\mathrm{mM} \mathrm{MgCl} 2$. Then the cells were co-incubated with $10 \mu \mathrm{M}$ of DMNDC and $300 \mathrm{nM}$ of MitoTracker Red/ LysoTracker Red/ ER Tracker Red for 15 minutes and washed with PBS $(\mathrm{pH} 7.4)$ twice before imaging.
DMNDC Cytotoxicity assay. Around 5,000 cells per well were seeded in a 96 well-plate and grown for $24 \mathrm{~h}$ in the aforementioned condition. After that, DMNDC was added from a DMSO stock. The added amount of DMSO was not more than $2 \mu \mathrm{L}$. After $24 \mathrm{~h}$ of incubation, $20 \mu \mathrm{L}$ of MTT dye solution (from a stock of $5 \mathrm{mg} / \mathrm{mL}$ in PBS buffer) was added to each well and incubated for $4 \mathrm{~h}$. The media was removed gently from each well, and $200 \mu \mathrm{L}$ DMSO was added to each well to dissolve the purple color crystal (30 min). The absorption at 570 $\mathrm{nm}$ was recorded in a Synergy $\mathrm{H} 1$ Hybrid Multi-mode microplate reader.

Confocal Microscopy: All the confocal images were acquired using Olympus FV-3000 confocal microscope with a live-cell imaging set up. For fluorescence imaging, 405, 488 and 561 $\mathrm{nm}$ excitation lasers were used. For $405,488 \mathrm{~nm}$ and $561 \mathrm{~nm}$ excitation, the emission windows were kept at $415-450 \mathrm{~nm}$, $500-560 \mathrm{~nm}$ and $565-650 \mathrm{~nm}$, respectively. The confocal aperture was kept at 0.81 Airy Disk (AU) while the dwell time was $8 \mu \mathrm{s} /$ pixel. The acquired images were processed using cellSens Dimension software.

\section{ACKNOWLEDGMENT}

We thank IISER Bhopal and SERB for financial support, DSTFIST facility at IISER Bhopal for the TEM images, and Prof. Samir Maji, IIT Bombay for the generous gift of $\alpha$-synuclein. IS is a recipient of the Ramanujan Fellowship from the Science and Engineering Research Board, India.

\section{REFERENCES}

(1) Rambaran, R. N.; Serpell, L. C. Amyloid Fibrils: Abnormal Protein Assembly. Prion 2008, 2 (3), 112-117.

(2) Glenner, G. G.; Wong, C. W. Alzheimer's Disease: Initial Report of the Purification and Characterization of a Novel Cerebrovascular Amyloid Protein. Biochem. Biophys. Res. Commun. 1984, 120 (3), 885-890.

(3) Jankovic, J. Parkinson's Disease: Clinical Features and Diagnosis. J. Neurol. Neurosurg. Psychiatry 2008, 79 (4), 368376.

(4) Dische, F. E.; Wernstedt, C.; Westermark, G. T.; Westermark, P.; Pepys, M. B.; Rennie, J. A.; Gilbey, S. G.; Watkins, P. J. Insulin as an Amyloid-Fibril Protein at Sites of Repeated Insulin Injections in a Diabetic Patient. Diabetologia 1988, 31 (3), 158161.

(5) Liu, R.; He, M.; Su, R.; Yu, Y.; Qi, W.; He, Z. Insulin Amyloid Fibrillation Studied by Terahertz Spectroscopy and Other Biophysical Methods. Biochem. Biophys. Res. Commun. 2010, 391 (1), 862-867.

(6) Patel, H. R.; Pithadia, A. S.; Brender, J. R.; Fierke, C. A.; Ramamoorthy, A. In Search of Aggregation Pathways of IAPP and Other Amyloidogenic Proteins: Finding Answers through NMR Spectroscopy. J. Phys. Chem. Lett. 2014, 5 (11), 1864 1870

(7) Rubin, N.; Perugia, E.; Goldschmidt, M.; Fridkin, M.; Addadi, L. Chirality of Amyloid Suprastructures. J. Am. Chem. Soc. 2008, 130 (14), 4602-4603.

(8) Nettleton, E. J.; Tito, P.; Sunde, M.; Bouchard, M.; Dobson, C. M.; Robinson, C. V. Characterization of the Oligomeric States of Insulin in Self-Assembly and Amyloid Fibril Formation by Mass Spectrometry. Biophys. J. 2000, 79 (2), 1053-1065.

(9) Domike, K. R.; Donald, A. M. Thermal Dependence of Thermally Induced Protein Spherulite Formation and Growth: Kinetics of $\beta$-Lactoglobulin and Insulin. Biomacromolecules 2007, 8 (12), 3930-3937.

(10) Pratim Bose, P.; Chatterjee, U.; Xie, L.; Johansson, J.; Göthelid, E.; Arvidsson, P. I. Effects of Congo Red on A $\beta 1-40$ Fibril Formation Process and Morphology. ACS Chem. Neurosci. 2010, 1 (4), 315-324.

(11) Manno, M.; Craparo, E. F.; Podesta, A.; Bulone, D.; Carrotta, R.; Martorana, V.; Tiana, G.; San Biagio, P. L. Kinetics of Different Processes in Human Insulin Amyloid Formation. $J$. Mol. Biol. 2007, 366 (1), 258-274.

(12) Bekard, I. B.; Dunstan, D. E. Tyrosine Autofluorescence as a 
Measure of Bovine Insulin Fibrillation. Biophys. J. 2009, 97 (9), 2521-2531.

(13) LeVine, H. 3rd. Thioflavine $T$ Interaction with Synthetic Alzheimer's Disease Beta-Amyloid Peptides: Detection of Amyloid Aggregation in Solution. Protein Sci. 1993, 2 (3), 404 410 .

(14) Groenning, M.; Norrman, M.; Flink, J. M.; van de Weert, M.; Bukrinsky, J. T.; Schluckebier, G.; Frokjaer, S. Binding Mode of Thioflavin T in Insulin Amyloid Fibrils. J. Struct. Biol. 2007, 159 (3), 483-497.

(15) Rodríguez-Rodríguez, C.; Rimola, A.; Rodríguez-Santiago, L.; Ugliengo, P.; Álvarez-Larena, Á.; Gutiérrez-de-Terán, H.; Sodupe, M.; González-Duarte, P. Crystal Structure of Thioflavin-T and Its Binding to Amyloid Fibrils: Insights at the Molecular Level. Chem. Commun. 2010, 46 (7), 1156-1158.

(16) Hong, Y.; Meng, L.; Chen, S.; Leung, C. W. T.; Da, L.-T.; Faisal, M.; Silva, D.-A.; Liu, J.; Lam, J. W. Y.; Huang, X.; et al. Monitoring and Inhibition of Insulin Fibrillation by a Small Organic Fluorogen with Aggregation-Induced Emission Characteristics. J. Am. Chem. Soc. 2012, 134 (3), 1680-1689.

(17) Suzuki, Y.; Brender, J. R.; Hartman, K.; Ramamoorthy, A.; Marsh, E. N. G. Alternative Pathways of Human Islet Amyloid Polypeptide Aggregation Distinguished by (19)f Nuclear Magnetic Resonance-Detected Kinetics of Monomer Consumption. Biochemistry 2012, 51 (41), 8154-8162.

(18) Gade Malmos, K.; Blancas-Mejia, L. M.; Weber, B.; Buchner, J.; Ramirez-Alvarado, M.; Naiki, H.; Otzen, D. ThT 101: A Primer on the Use of Thioflavin $\mathrm{T}$ to Investigate Amyloid Formation. Amyloid 2017, 24 (1), 1-16.

(19) Huang, H.; Li, P.; Zhang, M.; Yu, Y.; Huang, Y.; Gu, H.; Wang, C.; Yang, Y. Graphene Quantum Dots for Detecting Monomeric Amyloid Peptides. Nanoscale 2017, 9 (16), 5044-5048.

(20) Leung, C. W. T.; Guo, F.; Hong, Y.; Zhao, E.; Kwok, R. T. K.; Leung, N. L. C.; Chen, S.; Vaikath, N. N.; El-Agnaf, O. M.; Tang, Y.; et al. Detection of Oligomers and Fibrils of $\alpha$ Synuclein by AIEgen with Strong Fluorescence. Chem. Commun. 2015, 51 (10), 1866-1869.

(21) Li, Z.; Wang, H.; Chen, Z. Monitoring and Modulation of Insulin Fibers by a Protein Isomerization Targeting Dye Bromophenol Blue. Sensors Actuators B Chem. 2019, 287, 496502.

(22) Vázquez, M. E.; Blanco, J. B.; Imperiali, B. Photophysics and Biological Applications of the Environment-Sensitive Fluorophore 6-N,N-Dimethylamino-2,3-Naphthalimide. J. Am. Chem. Soc. 2005, 127 (4), 1300-1306.

(23) Sainlos, M.; Imperiali, B. Synthesis of Anhydride Precursors of the Environment-Sensitive Fluorophores 4-DMAP and 6-DMN. Nat. Protoc. 2007, 2 (12), 3219-3225.

(24) Mallick, S.; Pal, K.; Koner, A. L. Probing Microenvironment of Micelle and Albumin Using Diethyl 6(Dimethylamino)Naphthalene-2,3-Dicarboxylate: An Electroneutral Solvatochromic Fluorescent Probe. J. Colloid Interface Sci. 2016, 467, 81-89.

(25) Ratha, B. N.; Ghosh, A.; Brender, J. R.; Gayen, N.; Ilyas, H.; Neeraja, C.; Das, K. P.; Mandal, A. K.; Bhunia, A. Inhibition of Insulin Amyloid Fibrillation by a Novel Amphipathic Heptapeptide: MECHANISTIC DETAILS STUDIED BY SPECTROSCOPY IN COMBINATION WITH MICROSCOPY. J. Biol. Chem. 2016, 291 (45), 23545-23556.

(26) Nielsen, L.; Khurana, R.; Coats, A.; Frokjaer, S.; Brange, J.; Vyas, S.; Uversky, V. N.; Fink, A. L. Effect of Environmental Factors on the Kinetics of Insulin Fibril Formation: Elucidation of the Molecular Mechanism. Biochemistry 2001, 40 (20), 6036-6046.

(27) Li, S.; Leblanc, R. M. Aggregation of Insulin at the Interface. $J$. Phys. Chem. B 2014, 118 (5), 1181-1188.

(28) Mishra, N. K.; Joshi, K. B.; Verma, S. Inhibition of Human and Bovine Insulin Fibril Formation by Designed Peptide Conjugates. Mol. Pharm. 2013, 10 (10), 3903-3912.

(29) Li, Y.; Zhao, C.; Luo, F.; Liu, Z.; Gui, X.; Luo, Z.; Zhang, X.; Li, D.; Liu, C.; Li, X. Amyloid Fibril Structure of AlphaSynuclein Determined by Cryo-Electron Microscopy. Cell Res. 2018, 28 (9), 897-903.

(30) Knowles, T. P. J.; Vendruscolo, M.; Dobson, C. M. The Amyloid State and Its Association with Protein Misfolding
Diseases. Nat. Rev. Mol. Cell Biol. 2014, 15 (6), 384-396.

LaFerla, F. M.; Green, K. N.; Oddo, S. Intracellular Amyloid- $\beta$ in Alzheimer's Disease. Nat. Rev. Neurosci. 2007, 8 (7), 499 509 .

(32) Kinoshita, A.; Fukumoto, H.; Shah, T.; Whelan, C. M.; Irizarry, M. C.; Hyman, B. T. Demonstration by FRET of BACE Interaction with the Amyloid Precursor Protein at the Cell Surface and in Early Endosomes. J. Cell Sci. 2003, 116 (16), 3339 LP - 3346.

(33) Caspersen, C.; Wang, N.; Yao, J.; Sosunov, A.; Chen, X.; Lustbader, J. W.; Xu, H. W.; Stern, D.; McKhann, G.; Yan, S. $\mathrm{Du}$. Mitochondrial A $\beta$ : A Potential Focal Point for Neuronal Metabolic Dysfunction in Alzheimer's Disease. FASEB J. $\mathbf{2 0 0 5}$ 19 (14), 2040-2041.

(34) Wang, X.; Su, B.; Siedlak, S. L.; Moreira, P. I.; Fujioka, H.; Wang, Y.; Casadesus, G.; Zhu, X. Amyloid- $\beta$ Overproduction Causes Abnormal Mitochondrial Dynamics via Differential Modulation of Mitochondrial Fission/Fusion Proteins. Proc. Natl. Acad. Sci. 2008, 105 (49), 19318 LP - 19323.

(35) Singh, P. K.; Kotia, V.; Ghosh, D.; Mohite, G. M.; Kumar, A.; Maji, S. K. Curcumin Modulates $\alpha$-Synuclein Aggregation and Toxicity. ACS Chem. Neurosci. 2013, 4 (3), 393-407.

(36) Forli, S.; Huey, R.; Pique, M. E.; Sanner, M. F.; Goodsell, D. S.; Olson, A. J. Computational Protein-Ligand Docking and Virtual Drug Screening with the AutoDock Suite. Nat. Protoc. 2016, 11 (5), 905-919.

(37) Berman, H. M.; Westbrook, J.; Feng, Z.; Gilliland, G.; Bhat, T. N.; Weissig, H.; Shindyalov, I. N.; Bourne, P. E. The Protein Data Bank. Nucleic Acids Res. 2000, 28 (1), 235-242.

(38) BIOVIA, D. S. BIOVIA Discovery Studio Visualizer, V16. 1.0 15350, San Diego: Dassault Systemes; 2015 [Cited: 2017 Mar 20]. 
